Impacto de la endoglina y de otros marcadores de angiogénesis en las varices recurrentes. Resultados preliminaries

Impact of endoglin and other angiogenesis markers on recurrent varicose veins. Preliminary results 


\section{OR 353}

Impacto de la endoglina y de otros marcadores de angiogénesis en las varices recurrentes. Resultados preliminaries

Impact of endoglin and other angiogenesis markers on recurrent varicose veins. Preliminary results

José Antonio Carnicero Martínez ${ }^{1-3}$, Lucía Méndez García ${ }^{3,4}$, Miguel Pericacho Bustos ${ }^{3,4}$, Begoña García Cenador ${ }^{2,3}$, Francisco S. Lozano Sánchez ${ }^{1-3}$

${ }^{1}$ Servicio de Angiología, Cirugía Vascular y Endovascular y ${ }^{2}$ Departamento de Cirugía. Hospital Universitario de Salamanca. ${ }^{3}$ Instituto de Investigación Biomédica de Salamanca (IBSAL). ${ }^{4}$ Departamento de Fisiología y Farmacología. Hospital Universitario de Salamanca. Salamanca

Recibido: 02/09/2021

Aceptado: 03/09/2021

Correspondencia: Francisco S. Lozano Sánchez. Servicio de Angiología, Cirugía Vascular y Endovascular. Hospital Universitario de Salamanca. Paseo de San Vicente, s/n. 37007 Salamanca e-mail: lozano@usal.es

DOI: $10.20960 /$ angiologia.00353

Conflictos de interés: los autores declaran no tener ningún conflicto de interés.

Financiación: proyecto financiado por la Gerencia Regional de Salud de la Comunidad de Castilla y León (GRS 2047/A/19). 
Contribución de autores: concepción y diseño: F.S.L.S, J.A.C.M y M.P.B. Recolección de datos: J.A.C.M. y L.M.G. Análisis e interpretación: L.M.G., M.P.B., B.G.C. y F.S.L.S. Escritura del artículo: F.S.L.S. y M.P.B. Revisión crítica del artículo: J.A.C.M., M.P.B., B.G.C. y F.S.L.S. Aprobación final del artículo: J.A.C.M., L.M.G., M.P.B., B.G.C. y F.S.L.S. Análisis estadístico: M.P.B. y L.M.G.

\section{RESUMEN}

Introducción: después de una cirugía convencional de las varices (crosectomía y stripping de la safena interna) existe un importante porcentaje de recidivas que ocasiona un problema clínico, social y económico. Su etiopatogenia no está bien esclarecida.

Objetivo: investigar en pacientes con varices (no recidivadas y recidivadas) la expresión de diferentes moléculas implicadas en los procesos de neovascularización, inflamación e hipoxia.

Métodos: 41 pacientes sometidos a cirugía convencional de varices divididos en dos grupos: varices no recidivadas $(n=24)$ y varices recidivadas $(n=17)$. En ambos grupos se determinaron en la pared de la vena safena interna a nivel del cayado safenofemoral mediante RT-PCR a tiempo real los siguientes marcadores angiogénicos: endoglina, factor de crecimiento del endotelio vascular (VEGF-A), sus receptores 1 y 2 (VEGFR1 o FLTI), (VEGFR2 o KDR) y el factor inducible por hipoxia (HIF-1A). Todos los pacientes firmaron por escrito un consentimiento para participar en el estudio.

Resultados: en los pacientes con recidiva varicosa se aprecia, a nivel de la pared venosa, una superior expresión de endoglina $(p<0,05)$, VEGF-A $(p<0,05), \operatorname{FLT1}(p<0,001)$ y $\operatorname{KDR}(p<0,001)$ respecto del grupo de varices no recidivadas. No se observaron diferencias significativas respecto a HIF-1A.

Conclusión: existe un aumento de los marcadores de angiogénesis, incluida la endoglina, en las muestras de pacientes portadores de varices con recidivas. 
Palabras clave: Varices. Varices recurrentes. Angiogénesis. Endoglina.

\section{ABSTRACT}

Introduction: after conventional surgery for varicose veins (crosectomy and stripping of the internal saphenous) there is a significant percentage of recurrences which cause a clinical, social and economic problem. Its etiopathogenesis is not clear.

Objective: to investigate in patients with varicose veins (nonrecurrent and recurrent), the expression of different molecules involved in the processes of neovascularization, inflammation and hypoxia.

Methods: 41 patients undergoing conventional varicose vein surgery, divided into two groups: non-recurrent varicose veins $(n=24)$ and recurrent varicose veins $(n=17)$. In both groups, the following angiogenic markers were determined in the wall of the internal saphenous vein at the level of the saphenous-femoral arch, by means of real-time RT-PCR: endoglin, vascular endothelial growth factor (VEGF-A), its receptors 1 and 2 (VEGFR1 or FLTI), (VEGFR2 or KDR), and hypoxia-inducible factor (HIF-1A). All patients signed a written consent to participate in the study.

Results: in patients with varicose recurrence, a higher expression of endoglin $(p<0.05)$, VEGF-A $(p<0.05)$, FLT1 $(p<0.001)$ and KDR $(p<0.001)$ is appreciated in respect to the group of non-recurrent varicose veins. No significant differences were observed with respect to HIF-1A.

Conclusion: there is an increase in angiogenesis markers, including endoglin, in samples from patients with recurrent varicose veins.

Keywords: Varicose veins. Recurrent varicose veins. Angiogenesis. Endoglin. 


\section{INTRODUCCIÓN}

La enfermedad varicosa (EV) presenta elevada prevalencia en la población (30\%) (1), y ocasiona importantes problemas clínicos, sociales y económicos (2-4).

La EV forma parte del top 10 de las cirugías más frecuentes (5-6). La magnitud se incrementa porque una de cada cuatro consultas lo son por EV recurrente (EVR). La incidencia de EVR oscila entre el 13-65\%, según la técnica empleada (7); después de ligadura safenofemoral llega al $60 \%$ (8). Las reintervenciones son más complejas técnicamente, ocupan más tiempo aportan peores resultados y, además, empeoran la satisfacción del paciente e incrementan los costes globales.

Existen tres tipos de EVR: residuales (se "dejaron" en la intervención), verdadera EVR y nuevas varices (progresión de la enfermedad) (7).

Hasta hace poco se pensaba que las EVR se debían a procedimientos practicados inadecuadamente que dejaban remanentes de la safena o sus colaterales y que con el tiempo se desarrollaban varices recurrentes. Sin embargo, actualmente está aceptado que las causas son múltiples y en ocasiones combinadas (9). Así, se clasifican en: errores tácticos, errores técnicos, neovascularización y progresión de la enfermedad.

Un buen diagnóstico preoperatorio y una depurada técnica quirúrgica solo pueden prevenir errores tácticos y técnicos, cuando las otras causas suponen más del $50 \%$ de las EVR. Mientras que en el 10-35\% de las ocasiones la causa de recurrencia no se identifica, en el 50 $70 \%$ de los casos las recidivas suelen estar localizadas en la ingle (unión safenofemoral), y en ellas, la neovascularización es tan frecuente como el error táctico/técnico $(7,10)$.

Si la neovascularización es un factor de EVR tras cirugía, en las venas varicosas recidivadas podría mantenerse una mayor expresión de marcadores de angiogénesis que implicaría que se ha producido neovascularización angiogénica y que existe un mantenimiento de 
dicho proceso. El objetivo de este estudio es investigar, en estos pacientes, la expresión de diferentes moléculas implicadas en los procesos de neovascularización, inflamación e hipoxia.

\section{MATERIAL Y MÉTODO}

El presente estudio clínico prospectivo, observacional, abierto, controlado y no aleatorizado se ha realizado durante el periodo 20192020 en el Servicio de Angiología y Cirugía Vascular del Hospital Universitario de Salamanca. En la figura 1 se muestra el esquema de la investigación.

\section{Pacientes}

Criterios de inclusión: varices esenciales, hombres y mujeres de entre 18 y 70 años, ASA I-II, CEAP 2-6, diagnosticados mediante eco Doppler de una insuficiencia del cayado safenofemoral, sometidos a una crosectomía (ligadura/sección del cayado safenofemoral) y stripping de la safena y que firman el consentimiento para participar en el estudio.

Criterios de exclusión: varices postrombóticas y congénitas, mujeres embarazadas o parto en el último año, obesidad (IMC $>30 \mathrm{~kg} / \mathrm{m}^{2}$ ), ASA $\geq$ III, CEAP 0-1, no eco Doppler, otras técnicas quirúrgicas (por ejemplo, radiofrecuencia) y no consentimiento.

\section{Grupos}

- Varices no recidivadas (grupo 1): pacientes con varices esenciales e insuficiencia safenofemoral. Intervenidos mediante crosectomía y stripping de la safena interna.

- Varices recidivadas (grupo 2): pacientes con varices esenciales recidivadas a nivel safenofemonal. Reintervenidos con datos ecográficos preoperatorios sugestivos de neocayado.

\section{Variables a estudio}

- Cuaderno de registro de datos. 
- Determinación de cinco genes en tejidos: endoglina, factor de crecimiento del endotelio vascular (VEGF-A), receptores del VEGF (VEGFR1 o FLT1 y VEGFR2 o KDR) y HIF-1A (factor inducible por hipoxia).

\section{Recogida de muestras, procesamiento y almacenamiento}

Durante la cirugía, se obtuvo un fragmento de vena safena interna a nivel del cayado safenofemoral. Esta se introdujo inmediatamente en nitrógeno líquido y se almacenó a $-80^{\circ} \mathrm{C}$.

\section{Análisis de muestras}

Análisis de la expresión génica por RT-qPCR: analizamos la expresión de genes relacionados con la angiogénesis en muestras de tejido. Para ello, se pulverizó la muestra de tejido criogenizada y se extrajo su ARN. Posteriormente, se llevó a cabo la retrotranscripción de dicho ARN para poder obtener el ADNc, a partir del cual se estudia la expresión génica mediante PCR a tiempo real.

1. Extracción de ARN: para extraer el ARN de los tejidos obtenidos en la cirugía se utilizó el equipo comercial NucleoSpin ${ }^{\circledR}$ RNA (Macherey-Nagel). Para la extracción de ARN de los tejidos primero se pulveriza el tejido previamente criogenizado, se pesan $20 \mathrm{mg}$ de tejido y se lisan con el reactivo RA1 suplementado con $\beta$-mercaptoetanol al $1 \%$ utilizando unos micropistilos Kimble $^{\mathrm{TM}}$ Kontes $^{\mathrm{TM}}$ Pellet Pestle $^{\mathrm{TM}}$. Una vez lisada la muestra, se siguen las recomendaciones del fabricante. EI ARN obtenido se cuantifica empleando un espectrofotómetro NanoDrop ${ }^{\circledR}$ ND-1000 y se almacena a $-80{ }^{\circ} \mathrm{C}$.

2. Obtención de ADNc: para el análisis de la expresión génica, es necesario utilizar ADN como sustrato de la reacción, por lo que hay que transformar el ARN en ADN mediante retrotranscripción o transcripción reversa. Las transcriptasas reversas virales, descubiertas en 1970, sintetizan ADNc a partir de ARN. Con el 
fin de sintetizar ADNc, se diluyen $250 \mathrm{ng}$ de ARN de cada muestra con agua ultrapura hasta un volumen final de $16 \mu \mathrm{L}$. Después se añaden $4 \mu \mathrm{L}$ de la iScript ${ }^{\mathrm{TM}}$ Reverse Transcription Supermix (BioRad) $5 X$ y se incuban en un termociclador MyCycler a $25{ }^{\circ} \mathrm{C}$ durante 5 minutos, seguidos de 30 minutos a $42{ }^{\circ} \mathrm{C}$ y 5 minutos finales a $85^{\circ} \mathrm{C}$. El ADNc obtenido se almacena a $-20^{\circ} \mathrm{C}$.

3. PCR cuantitativa, qPCR o PCR a tiempo real: la PCR se preparará sobre un volumen final de $20 \mu \mathrm{L}: 1 \mu \mathrm{L}$ de ADNc, $10 \mu \mathrm{L}$ de la Supermix $\mathrm{iQ}^{\circledR} \mathrm{SYBR}^{\circledR}$ Green (BioRad), 0,4 $\mu \mathrm{L}$ de cada cebador a una concentración de $20 \mathrm{mM}$ y $8,2 \mu \mathrm{L}$ de agua ultrapura. La reacción se lleva a cabo en un termociclador iQ ${ }^{\circledR} 5$ empleando un protocolo que incuba la reacción a $95{ }^{\circ} \mathrm{C}$ durante 5 minutos, seguidos de 40 ciclos de 30 segundos a $95^{\circ} \mathrm{C}, 30$ segundos a la temperatura de anillamiento óptima y 30 segundos a $72{ }^{\circ} \mathrm{C}$. La cámara acoplada al termociclador toma una imagen de la muestra al final de cada ciclo y detecta la señal fluorescente, que será más grande cuanto más producto de PCR haya.

Se utilizaron como controles o housekeeping la expresión de los ARNm de GAPDH.

\section{Análisis estadístico}

Los datos están expresados en un diagrama de caja (box plot). Para el análisis entre grupos se ha empleado la prueba $t$ de Student. El resultado estadístico es significativo a partir de 0,05.

\section{Responsabilidades éticas}

La investigación fue aprobada por el Comité Ético de Investigación del Área de Salud de Salamanca, que se ajusta a las normas éticas y de buena práctica clínica (de la Asociación Médica Mundial y de la Declaración de Helsinki). Código CEIm: PI 201903 204, aprobado el 1/3/2019. 
Todos los pacientes firmaron un consentimiento para participar en el estudio. Todos los datos se han tratado de forma confidencial y han sido encriptados según lo dispuesto en la Ley Orgánica 15/1999, de 13 de diciembre, de Protección de Datos de Carácter Personal y en la Ley 14/2007, de 3 de julio, de Investigación biomédica.

\section{RESULTADOS}

En el estudio se han incluido 41 pacientes divididos en dos grupos: varices no recidivadas $(n=24)$ y varices recidivadas $(n=17)$. El reparto por sexos y edades se ve en la tabla I.

El análisis de la expresión de marcadores de angiogénesis e hipoxia en las muestras de tejido obtenidas de los grupos demuestra que el grupo 2 (venas safenas de pacientes con recidiva) presenta una mayor expresión de endoglina $(p<0,05)$, VEGF-A $(p<0,05)$, FLT1 $(p<0,001)$ y $\operatorname{KDR}(p<0,001)$ respecto del grupo 1 (Fig. 2$)$. No se detectaron diferencias entre grupos respecto a la expresión del gen marcador de HIF1A. En la tabla II se muestran los datos numéricos por grupos.

\section{DISCUSIÓN}

En 1950 se definieron los principios del tratamiento quirúrgico de la EV (interrumpir el reflujo desde el sistema venoso profundo al superficial). Desde entonces, han sido muchos los avances diagnósticos (como, por ejemplo, el eco Doppler preoperatorio) y terapéuticos (como las técnicas endovasculares). Sin embargo, la EVR está por resolver. Persisten las controversias sobre qué técnica (stripping, radiofrecuencia, láser endovenoso, etc.) es más eficaz. Así, el estudio RECLAS atribuye iguales recidivas al láser y a la tradicional ligadura safenofemoral (stripping) (11). Un estudio posterior tampoco encontró diferencias relacionadas con las recidivas entre la cirugía convencional y la endovenosa (láser o radiofrecuencia), aunque las causas de recidiva son diferentes (12). 
De las cuatro causas de EVR descritas (9), la neovascularización es el mecanismo más recientemente implicado en la patogenia de las EVR. Una revisión concluye que la neovascularización es una causa común de recidiva varicosa poscirugía: empleando el eco Doppler, se observa del 25 al $94 \%$ de las EVR (7).

Nuestros resultados, aunque preliminares, apoyan que la neovascularización forma parte de la compleja etiopatogenia de la recidiva varicosa al constatar un significativo aumento de diversos marcadores de angiogénesis en los pacientes con EVR.

La neovascularización supone la formación de nuevos vasos sanguíneos, que en el caso de la EVR aparecen en posición anormal. Estos nuevos vasos, de diferente tamaño, número y tortuosidad, surgen por igual después de una cirugía convencional (stripping) o endovenosa (láser o radiofrecuencia), aunque es inferior en las técnicas de ablación endovenosa, dado que en estos procedimientos no existe derramamiento de células endoteliales, circunstancia que puede ser el origen de la neovascularización. De hecho, en estas técnicas, la recanalización es la causa más frecuente de EVR (12).

En la patogénesis de la neovascularización, como causa de EVR poscirugía, se contemplan factores intraoperatorios (técnica quirúrgica, trauma ocasionado, material de sutura, etc.) y posoperatorios (hipoxia, inflamación, moléculas proangiogénicas, etc.) (Fig. 3A y B).

Existen diferentes hipótesis. La más aceptada es que la hipoxia de la pared venosa, la neovascularización y la enfermedad varicosa esencial/recurrente están fuertemente relacionadas. La neovascularización se produce por el estímulo angiogénico en el área cicatricial de la ligadura de la unión safenofemoral y en la extracción del tronco safeno: se forman neovasos que reconectan con troncos venosos residuales recurrentes. Dicho de otra manera: puede ser una respuesta a la desconexión venosa, una respuesta cicatricial que, por la hipoxia, induce activación endotelial y liberación de factores angiogénicos que producen una recanalización y una endotelización 
del trayecto de la vena resecada. El mantenimiento de la señal angiogénica, caracterizada por la persistencia de marcadores angiogénicos en el neovaso, explicaría la compleja red tortuosa de neovasos que conectan con el muñón de la vena safena, con sus tributarias y con la vena femoral común (Fig. 4).

Aunque existen estudios observacionales que correlacionan los hallazgos del eco Doppler preoperatorio con la impresión macroscópica durante la reintervención y el estudio histológico de la pieza como sugestivos de neovascularización (13), la investigación debe focalizarse en los mecanismos fisiopatológicos que la producen tras una correcta intervención quirúrgica y, por ello, la atención debe dirigirse a moléculas diana involucradas en los procesos neoangiogénicos, tales como HIF, VEGF o endoglina (Fig. 3C).

El factor inducible por hipoxia (HIF) ha sido investigado en la patogénesis de diversas enfermedades vasculares arteriales (como la arteriosclerosis) y venosas, tanto agudas como crónicas (varices) (14). Es bien conocido que la activación del HIF está implicada en la angiogénesis. En nuestra investigación, el factor HIF-1, aunque aumentado respecto de un control (pacientes sin varices), no mostró diferencias significativas entre los grupos de pacientes con varices no recurrentes y recurrentes; no determinamos HIF-2. La ausencia de diferencias podría ser indicativa de que el vaso ya se ha formado y se ha recuperado el flujo de sangre en la zona.

Dentro de los marcadores de neovascularización se ha observado una mayor expresión de decorina en muñones redundantes (después de la ligadura del cayado safenofemoral) (8). Para estos autores, la decorina, una proteína del tejido conectivo, permite diferenciar entre muñón redundante y muñón redundante y neovascularización como causas de EVR. En otros marcadores empleados por estos autores (VEGF, TGF- $\beta 1$ y TGF- $\beta 3$, metaloproteinasa 1 , etc.) no se encontraron diferencias entre grupos. Por el contrario, nuestro estudio muestra incrementos significativos de VEGF-A, de sus receptores (FLT1 y KDR) y de endoglina (este último, correceptor de TGF- $\beta$ ). 
La endoglina es una glicoproteína de membrana que se expresa especialmente en las células endoteliales, participa en la señalización de diversas moléculas de la familia del TGF- $\beta$ y modula las respuestas celulares a TGF- $\beta$, entre ellas la producción de matriz extracelular, la regulación de la angiogénesis, el remodelado vascular y el desarrollo cardiovascular (15). La presencia de endoglina en las células de las tres capas de los vasos sanguíneos (íntima, media y adventicia) sugiere un importante papel en la fisiología vascular. Por su parte, TGF- $\beta$ actúa en la neoformación de capilares y en el mantenimiento de la integridad de la pared de los vasos, tanto en el embrión como en la vida posnatal $(15,16)$. Además de la forma de membrana, la estudiada por nosotros, existe una forma soluble de endoglina formada por procesamiento proteolítico de la isoforma de membrana (15).

Por todo esto, la endoglina como molécula proangiogénica es la clave en la regulación de la neoangiogénesis y es esencial para una correcta angiogénesis (16). Recientemente se ha propuesto que, al igual que es necesario un aumento de expresión de endoglina para el correcto desarrollo del proceso angiogénico, es necesaria una disminución de su expresión para que la angiogénesis pueda resolverse correctamente. La persistencia de la expresión de endoglina daría lugar a alteraciones vasculares por ese exceso de la angiogénesis.

Por otro lado, para confirmar que el grupo 2 tenía una mayor angiogénesis en la zona de la cirugía, analizamos también otros marcadores, como VEGF-A, que es un conocido e importante factor implicado en la angiogénesis, y sus receptores de tipo 1 (FLT1) y 2 (KDR). Los resultados obtenidos confirman que las varices procedentes de pacientes con recidiva tienen un aumento significativo de la expresión de todos estos genes relacionados con la angiogénesis. El esquema de actuación propuesto se representa en la figura 5 . 
El papel de la endoglina y su forma soluble no han sido investigados en las EVR. Únicamente existe una publicación que analiza el papel de TGF- $\beta$ y de la endoglina en la cicatrización de úlceras venosas (17).

Con la finalidad de reducir o de evitar la estimulación de la angiogénesis poscirugía venosa, se han ideado técnicas de barrera (cierre de la fascia cribiforme, cobertura del muñón con una prótesis de PTFE), la inversión del endotelio del muñón o técnicas de avulsión (18-20). La recomendación de estas técnicas es baja (21).

En este sentido, nuestro trabajo también busca un potencial impacto clínico. Los resultados de endoglina en tejidos son positivos. En una segunda fase investigaremos la endoglina soluble: como es factible determinarla en el plasma (no en tejidos), podría convertirse en un marcador predictivo de recurrencia varicosa.

Existen limitaciones en nuestro estudio. Primera, el escaso número de pacientes por grupo, motivado por la reducción de cirugías durante la pandemia por la COVID-19. Estos resultados preliminares se completarán con más casos ( $n=30$ por grupo). Segunda, el limitado número de moléculas analizadas está motivado por problemas de costes. Se seleccionaron aquellas no investigadas (endoglina) y otras que, aunque estudiadas, son fundamentales como patrones de referencia (VEGF o HIF).

\section{CONCLUSIONES}

Hemos detectado un aumento de marcadores de angiogénesis en los pacientes con varices recidivadas. Específicamente en la pared venosa de la safena a nivel safenofemoral de estos pacientes (frente al grupo sin recidivadas) existe una mayor y significativa expresión de endoglina, de VEGF-A y de sus receptores FLT1 y KDR. No encontramos diferencias respecto a HIF-1A.

\section{BIBLIOGRAFÍA}


1. Lozano FS, Masegosa A, Alvarez J, Marinello J; DETECT-2006 Group. Occurrence and management of chronic venous disease in primary health care in Spain. A comparison of DETECT-2006 with DETECT-2000. Int Angiol 2009;28(1):62-7.

2. Lozano Sánchez FS, Marinello Roura J, Carrasco Carrasco E, González-Porras JR, Escudero Rodríguez JR, Sánchez Nevárez I, et al. Venous leg ulcer in the context of chronic venous disease. Phlebology 2014(4);29:220-6. DOI: 10.1177/0268355513480489

3. Lozano Sánchez FS, Sánchez Nevárez I, González-Porras JR, Marinello Roura J, Escudero Rodríguez JR, Díaz Sánchez S, et al. Quality of life in patients with chronic venous disease: influence of the socio-demographical and clinical factors. Int Angiol 2013(4);32:433-41.

4. Lozano Sánchez FS, Asensi Pallarés E. Reclamaciones médicolegales y cirugía de varices. Peritaje de un cirujano vascular durante 25 años. Angiología 2016(4);68:292-7. DOI: 10.1016/j.angio.2015.10.015

5. Kanwar A, Hansrani M, Lees T, Stansby G. Trends in varicose vein therapy in England: radical changes in the last decade. Ann $R$ Coll Surg Engl 2010;92(4):341-6. DOI: 10.1308/003588410X12518836440649

6. Lozano FS, Marinello J, Moreno RM, Aguilar MD, López-Quintana A, González-Porras JR, et al. Monitoring the practice of vascular surgery: findings from a national registry (1996-2011). World J Surg 2014;38(1):241-51. DOI: 10.1007/s00268-013-2272-6

7. Brake M, Lim CS, Shepherd AC, Shalhoub J, Davies AH. Pathogenesis and etiology of recurrent varicose veins. J Vasc Surg 2013;57(3):860-8. DOI: 10.1016/j.jvs.2012.10.102

8. Reich-Schupke S, Mumme A, Altmeyer P, Stuecker M. Decorin expression with stump recurrence and neovascularization after varicose vein surgery-a pilot study. Dermatol Surg 2011(4);37:480-5. DOI: 10.1111/j.1524-4725.2011.01912.x

9. Kostas T, loannou CV, Touloupakis E, Daskalaki E, Giannoukas $A D$, Tsetis $D$, et al. Recurrent varicose veins after surgery: a new appraisal of a common and complex problem in vascular 
surgery. Eur J Vasc Endovasc Surg 2004;27(3):275-82. DOI: 10.1016/j.ejvs.2003.12.006

10. Perrin MR, Labropoulos N, Leon JL. Presentation of the patient with recurrent varices after surgery (REVAS). J Vasc Surg 2006;43(2):327-34. DOI: 10.1016/j.jvs.2005.10.053

11. Rass K, Frings N, Glowacki P, Gräber S, Tilgen W, Vogt T. Same site recurrence is more frequent after endovenous laser ablation compared with high ligation and stripping of the great saphenous vein: 5 year results of a randomized clinical trial (RELACS study). Eur J Vasc Endovasc Surg 2015;50(5):648-56. DOI: 10.1016/j.ejvs.2015.07.020

12. O'Donnell TF, Balk EM, Dermody M, Tangney E, lafrati MD. Recurrence of varicose veins after endovenous ablation of the great saphenous vein in randomized trials. J Vasc Surg Venous Lymphat Disord 2016;4(1):97-105. DOI: 10.1016/j.jvsv.2014.11.004

13. Van Rij AM, Jones GT, Hill GB, Jiang P. Neovascularization and recurrent varicose veins: more histologic and ultrasound evidence. J Vasc Surg 2004;40(2):296-302. DOI: 10.1016/j.jvs.2004.04.031

14. Lim CS, Kiriakidis S, Sandison A, Paleolog EM, Davies AH. Hypoxia-inducible factor pathway and diseases of the vascular wall. J Vasc Surg 2013;58(1):219-30. DOI: 10.1016/j.jvs.2013.02.240

15. Ollauri-Ibáñez C, López-Novoa JM, Pericacho M. Endoglinbased biological therapy in the treatment of angiogenesisdependent pathologies. Expert Opin Biol Ther 2017;17(9):105363. DOI: 10.1080/14712598.2017.1346607

16. Eleno N, López-Novoa JM, Lozano F. Endoglin: a marker of neoplasias or rather of neo-angiogenesis? Letter to the editor. Head Neck 2010(7);32:971. DOI: 10.1002/hed.21248

17. Ligi D, Croce L, Mosti G, Raffetto JD, Mannello F. Chronic venous insufficiency: Transforming growth factor- $\beta$ isoforms and soluble endoglin concentration in different states of wound healing. Int J Mol Sci 2017;18(10):2206. DOI: 10.3390/ijms18102206 
18. De Maeseneer MG, Philipsen TE, Vandenbroeck CP, Lauwers PR, Hendriks JM, De Hert SG, et al. Closure of the cribiform fascia: an efficient anatomical barrier against postoperative neovascularization at the saphenofemoral junction. A prospective study. Eur J Vasc Endovasc Surg 2007;34(3):361-6. DOI: 10.1016/j.ejvs.2007.03.020

19. an Rij AM, Jones GT, Hill BG, Amer M, Thomson IA, Pettigrew RA, et al. Mechanical inhibition of angiogenesis at the saphenofemoral junction in the surgical treatment of varicose veins: early results of a blinded randomized controlled trial. Circulation 2008;118(1):66-74.

DOI: 10.1161/CIRCULATIONAHA.107.726869

20. Kokubo M, Nozaka T, Takahashi Y. New method of flush saphenofemoral ligation that is expected to inhibit varicose vein recurrence in the groin: Flush ligation using the avulsion technique method. Ann Vasc Dis 2018;11(3):286-91. DOI: 10.3400/avd.oa.18-00086

21. Howard A, Howard DP, Davies AH. Surgical treatment of the incompetent saphenous vein. En: Peter Gloviczki P, editor. Handbook of venous disorders. Guidelines of the American Venous Forum. $3^{a}$ ed. London: Hodder Arnold; 2009. pp. 400-8.

Tabla I. Características básicas de los grupos

\begin{tabular}{|l|l|l|l|}
\hline Grupo & $\begin{array}{l}\text { N.o de } \\
\text { pacientes }\end{array}$ & Sexo & Edad \\
\hline $\begin{array}{l}\text { 1 (no } \\
\text { recidiva) }\end{array}$ & 24 & $\begin{array}{l}7 \text { hombres } \\
17 \\
\text { mujeres }\end{array}$ & 33-67 años \\
\hline 2 (recidiva) & 17 & $\begin{array}{l}8 \text { hombres } \\
9 \text { mujeres }\end{array}$ & 32-69 años \\
\hline
\end{tabular}

Tabla II. Expresión de genes relacionados con la angiogénesis en muestras de vena safena interna obtenidas durante la cirugía 


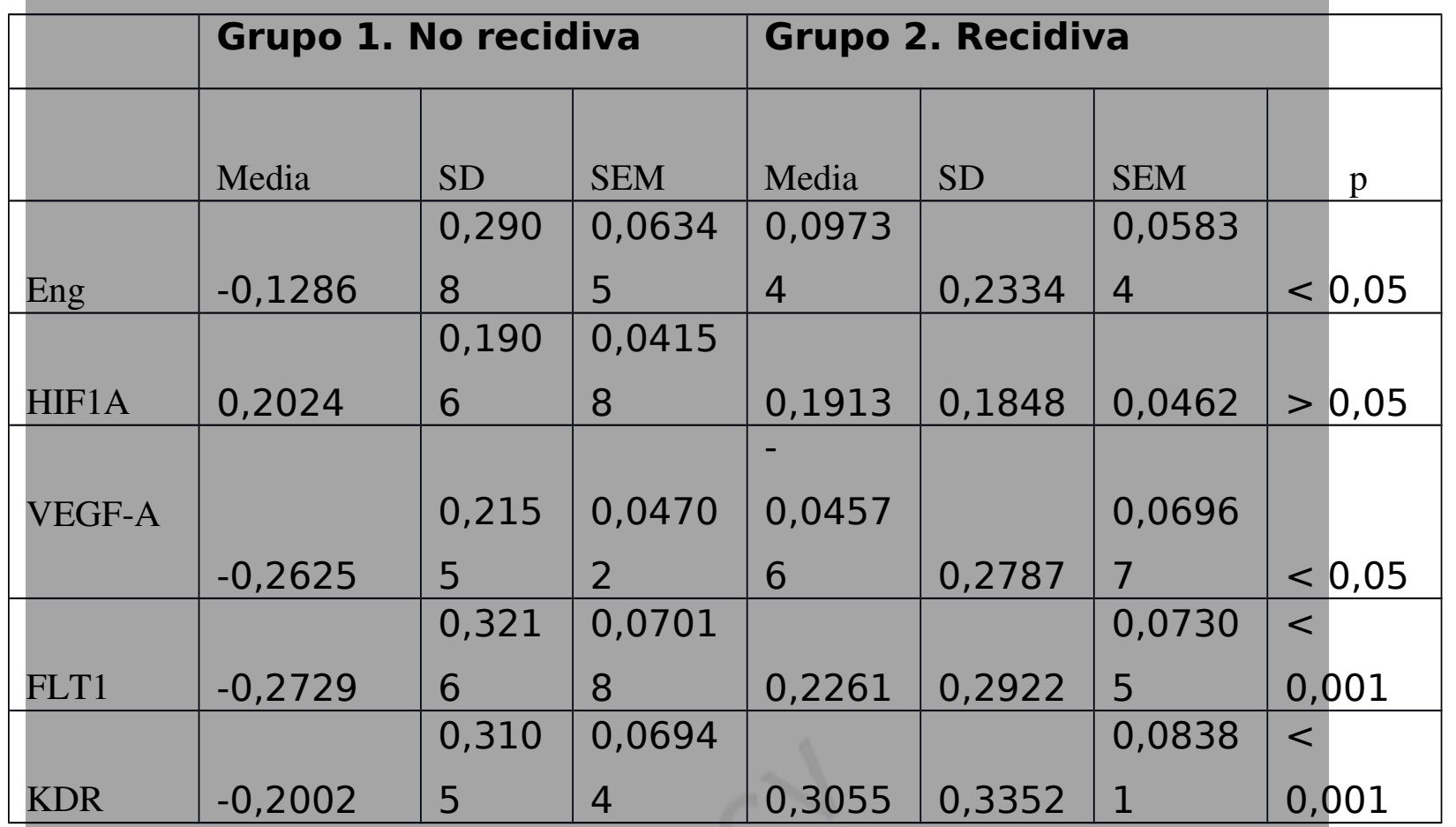

Eng: endoglina; HIF1A: factor inducible por hipoxia; VEGF-A: factor de crecimiento del endotelio vascular; FLTI (o VEGFR1): receptor 1 del VEGF; KDR (o VEGFR2): receptor 2 del VEGF.

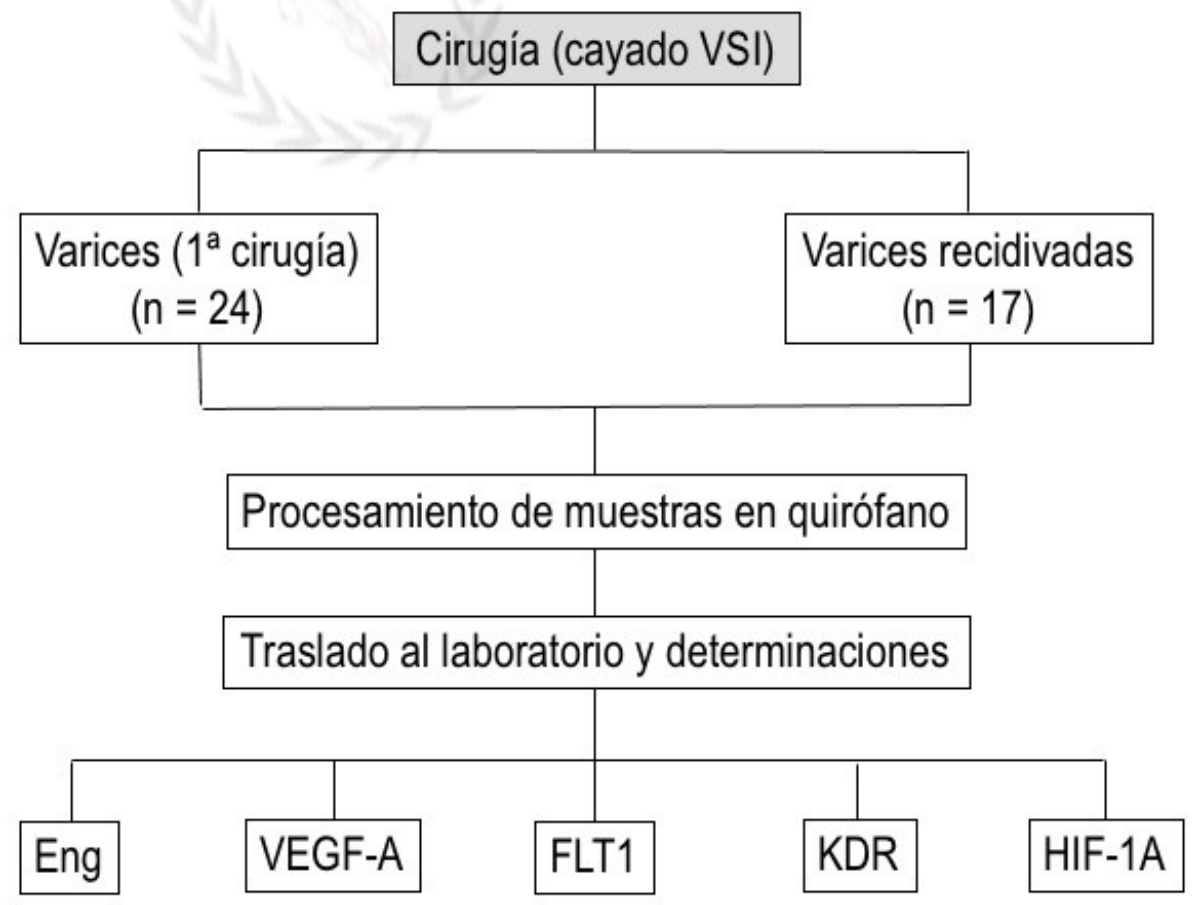

Figura 1. Esquema de la investigación. 
VSI: vena safena interna; Eng: endoglina; VEGF-A: factor de crecimiento del endotelio vascular; FLT1 y KDR: receptores 1 y 2 del VEGF; HIF-1A: factor inducible por hipoxia.
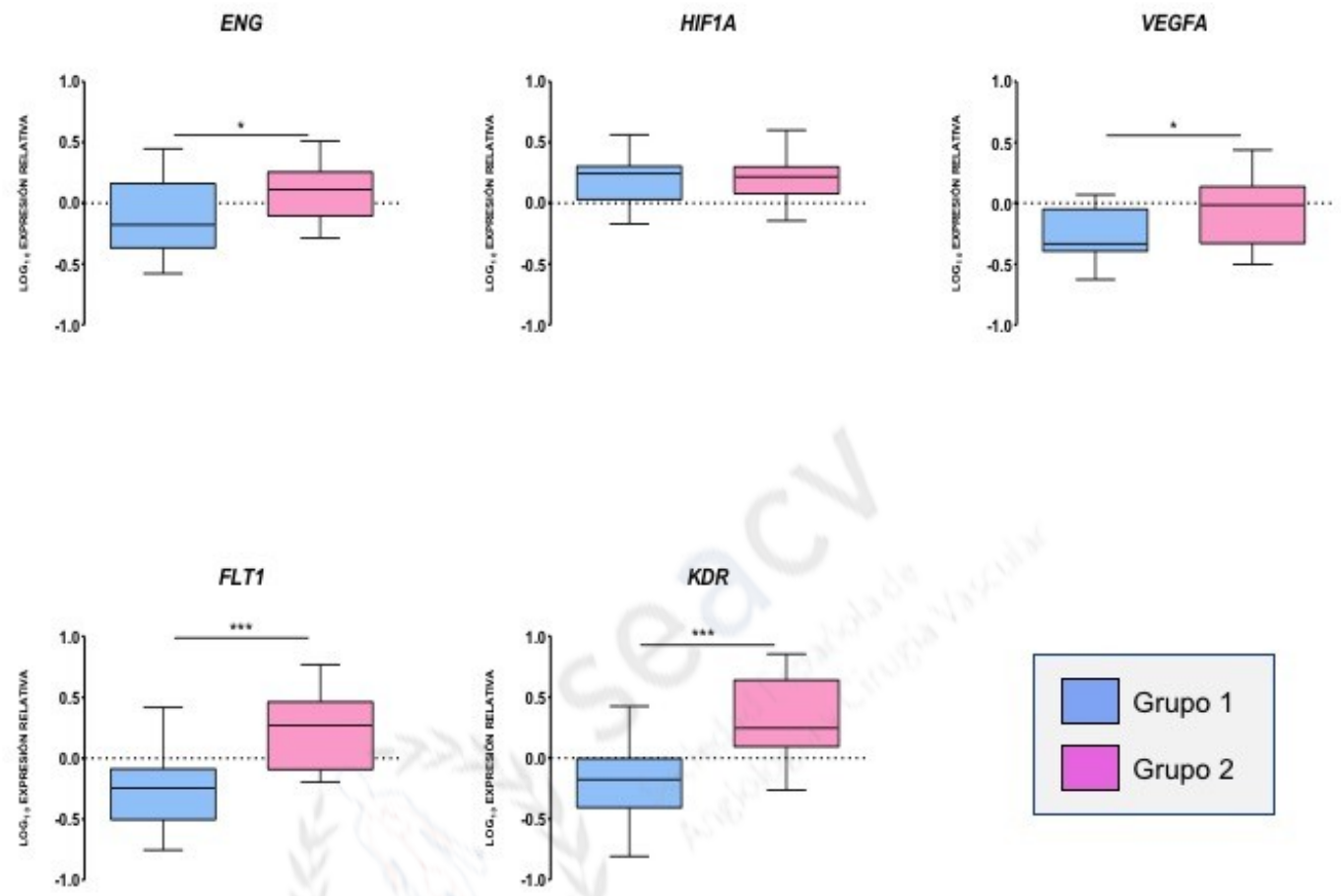

Figura 2. Expresiones de genes relacionados con la angiogénesis en muestras de vena safena interna obtenidas durante la cirugía.

ENG: endoglina; HIF1A: factor inducible por hipoxia; VEGF-A: factor de crecimiento del endotelio vascular; FLT1 y KDR: receptores 1 y 2 del VEGF. Significación estadística: *p $<0,05 ; * * * p<0,001$ 

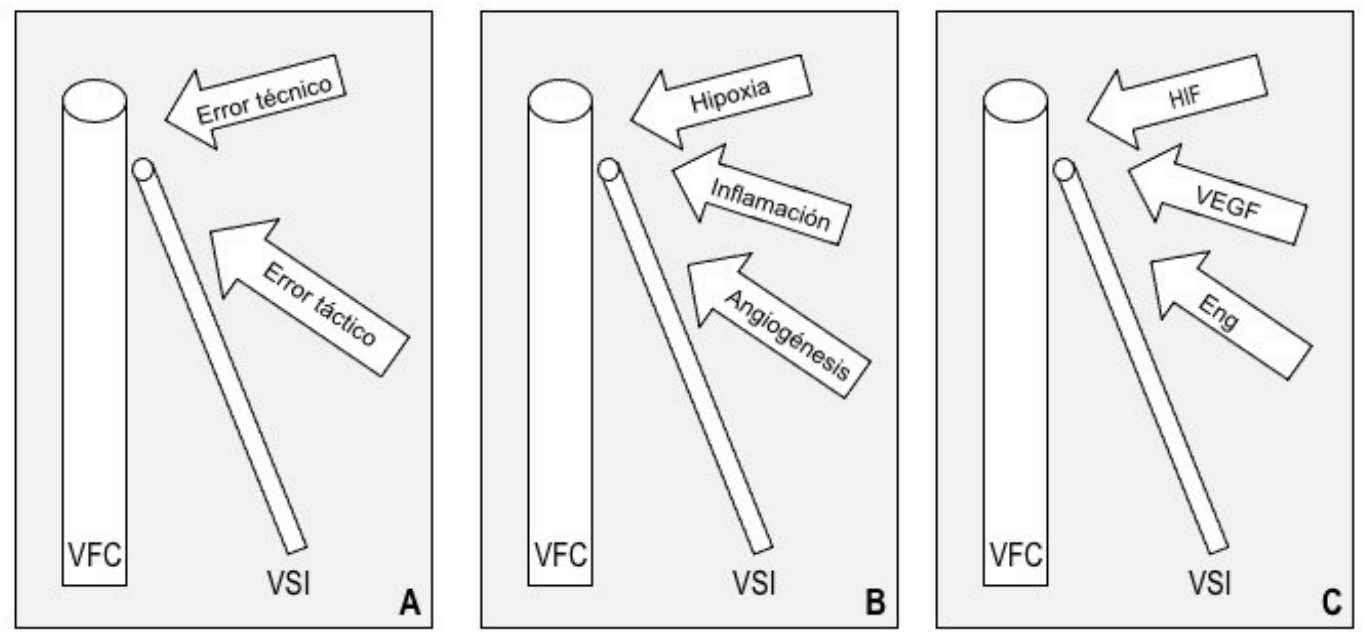

Figura 3. Patogénesis de las recurrencias varicosas. A. Mecanismos clásicos. B. Mecanismos actuales. C. Moléculas investigadas (hipótesis de nuestra investigación). VFC: vena femoral común; VSI: vena safena interna; HIF: factor inducible por hipoxia; VEGF: factor de crecimiento del endotelio vascular; Eng: endoglina.

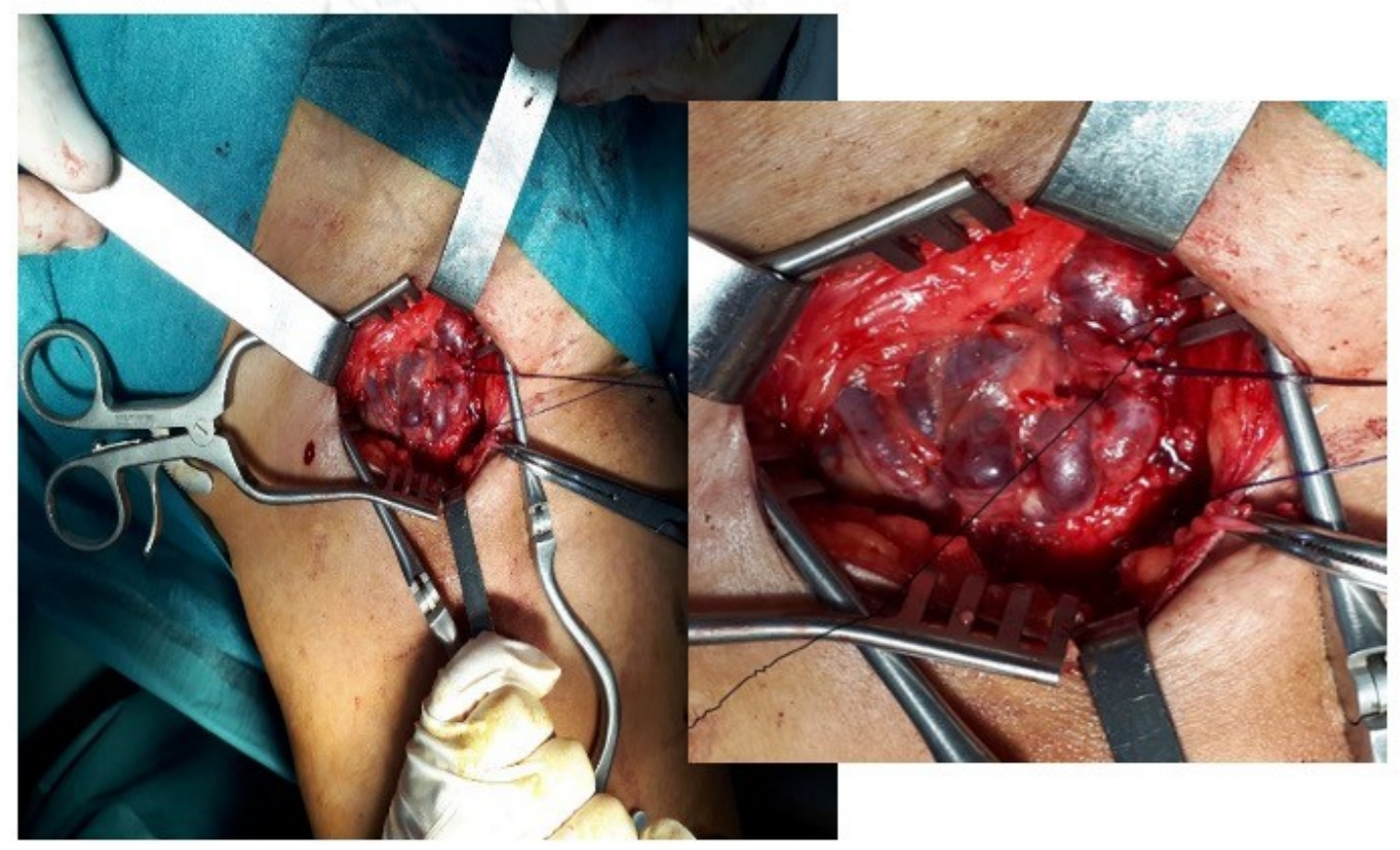

Figura 4. Imagen intraoperatoria de un neocayado a nivel inguinal. Detalle del ovillo probablemente neoangiogénico. 


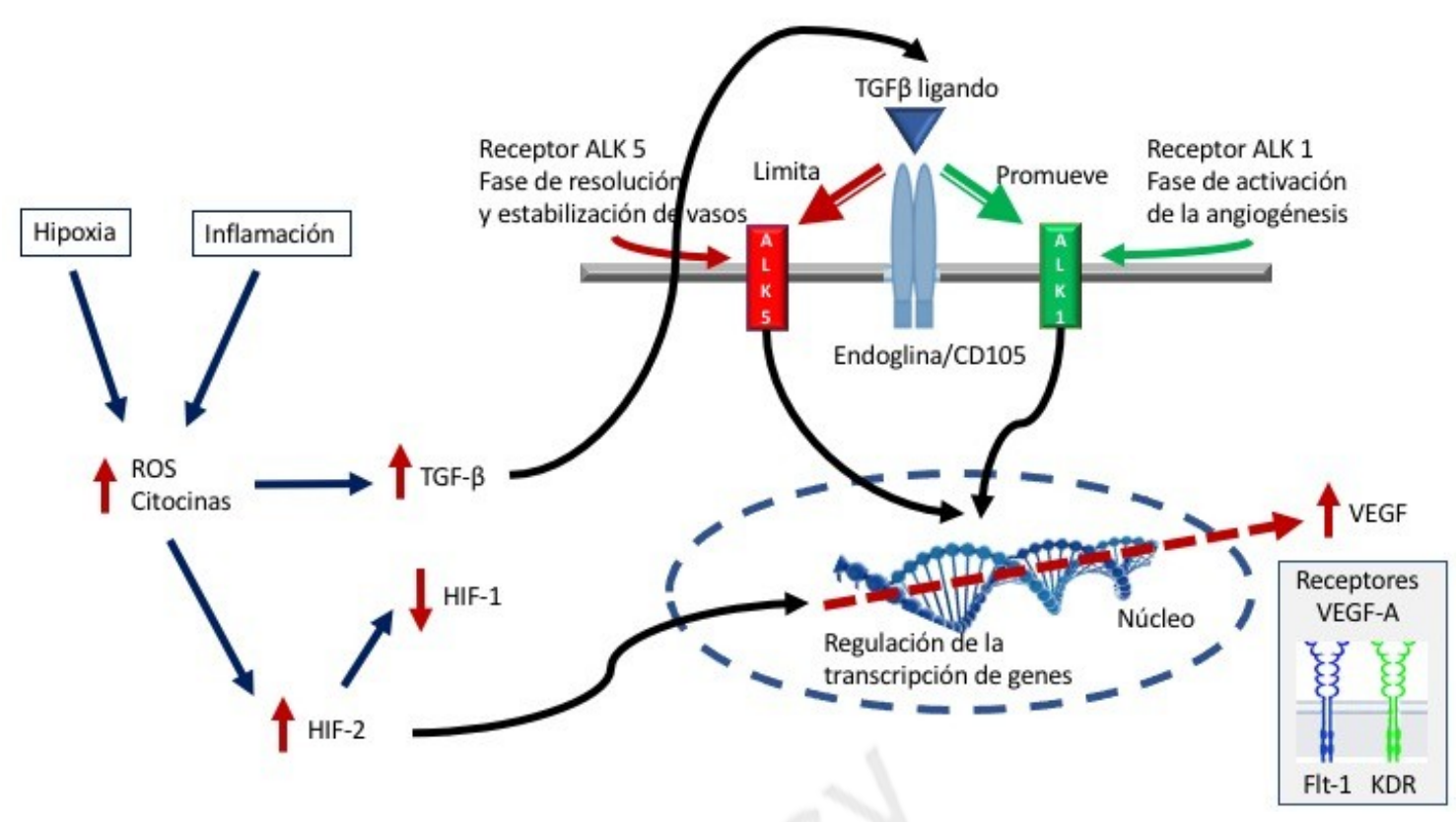

Figura 5. Factores moleculares posoperatorios que pueden contribuir a la recidiva de venas varicosas.

ROS: especies reactivas de oxígeno; HIF-1 y 2: factores inducibles por hipoxia 1 y 2; TGF- $\beta$ : factor de crecimiento transformante-beta; ALK 5 y 1: quinasas similar-receptor activina 5 y 1 ; endoglina/CD105: endoglina de membrana; VEGF: factor de crecimiento del endotelio vascular; FLT1 y KDR o receptores 1 y 2 del VEGF-A. 